\title{
BMJ Open Systematic review of the effectiveness of complementary and alternative medicine on nausea and vomiting in children with cancer: a study protocol
}

\author{
Ka Yan Ho, ${ }^{1}$ Katherine Ka Wai Lam, ${ }^{1}$ Joyce Oi Kwan Chung, ${ }^{1}$ Wei Xia, ${ }^{2}$ \\ Ankie Tan Cheung, ${ }^{2}$ Long Kwan Ho, ${ }^{2}$ Sau Ying Chiu, ${ }^{3}$ Godfrey Chi Fung Chan, ${ }^{4}$ \\ Ho Cheung William LI (1) ${ }^{2}$
}

To cite: Ho KY, Lam KKW, Chung JOK, et al. Systematic review of the effectiveness of complementary and alternative medicine on nausea and vomiting in children with cancer: a study protocol. BMJ Open 2019;9:e031834. doi:10.1136/ bmjopen-2019-031834

- Prepublication history and additional material for this paper are available online. To view these files, please visit the journal online (http://dx.doi. org/10.1136/bmjopen-2019031834).

Received 21 May 2019 Revised 24 September 2019 Accepted 24 September 2019

Check for updates

(C) Author(s) (or their employer(s)) 2019. Re-use permitted under CC BY-NC. No commercial re-use. See rights and permissions. Published by BMJ.

${ }^{1}$ School of Nursing, The Hong Kong Polytechnic University, Hong Kong, China

${ }^{2}$ School of Nursing, The University of Hong Kong, Hong Kong, China

${ }^{3}$ Department of Paediatrics \& Adolescent Medicine, Hong Kong Children's Hospital, Hong Kong, China

${ }^{4}$ Department of Paediatrics and Adolescent Medicine, The University of Hong Kong, Hong Kong, China

Correspondence to Dr Ho Cheung William Ll; william3@hku.hk

\section{ABSTRACT}

Introduction Nausea and vomiting are two most common symptoms reported by children with cancer when they undergo active treatment. However, pharmacological treatment is not sufficient to manage these two symptoms, with over $40 \%$ of children still experience nausea and vomiting after receiving antiemetics. There has been an exponential growth of studies to demonstrate the effectiveness of different complementary complementary medicine (CAM) to control nausea and vomiting during cancer treatment. Appropriate application of CAM enhances the effectiveness of antiemetics, thus reducing the symptom burden on children as well as improving their general condition and quality of life during cancer treatment. Nevertheless, it remains unclear which CAM is the best approach to help children to prevent or reduce nausea and vomiting during and after cancer treatment. This paper describes a protocol for identifying, analysing and synthesising research evidence on the effectiveness of CAM on nausea and vomiting in children with cancer.

Methods and analysis A total of 10 databases will be searched to identify appropriate literature: MEDLINE, the Cochrane Central Register of Controlled Trials (CENTRAL), EMBASE, CINAHL, PsycINFO, LILACS, OpenSIGLE, the Chinese Biomedical Literature Database, the Chinese Medical Current Contents and the Chinese National Knowledge Infrastructure. All randomised controlled trials which meet the inclusion criteria will be included. The primary outcome is the changes in nausea and vomiting either assessed by self-reported and/or objective measures. Review Manager 5.3 will be used to synthesise the data, calculate the treatment effects, perform any subgroup analysis and assess the risk of bias.

Ethical and dissemination The results will be presented at international conferences and published in peerreviewed journals. As no individual data will be involved in this review, ethical approval is not required.

PROSPERO registration number CRD42019135404.

\section{INTRODUCTION}

\section{Description of the condition}

Cancer is a leading cause of death in children, with an annual incidence of 300000 new cases worldwide. ${ }^{1}$ Because of breakthroughs
Strengths and limitations of this study

- This review will employ a systematic approach to evaluate the effectiveness of complementary and alternative therapies in reducing nausea and vomiting among children with cancer.

- Screening and selection of studies and data extraction and management will be performed by two independent researchers.

- Meta-analysis may not be performed due to insufficient homogeneous studies.

- There may be deficient in high-quality trials to generate convincing conclusions.

- Clinical characteristics will confound the results if a subgroup analysis cannot be performed.

in medical treatment, the survival rate for most types of childhood cancer has now increased to $>80 \% .^{2}$ Despite the improved survival, children with cancer still experience substantial adverse effects of cancer treatment. ${ }^{2-4}$ The two most commonly reported symptoms are nausea and vomiting. ${ }^{3}$ Nausea is a subjective experience characterised by a feeling of impending vomiting, ${ }^{5}$ whereas vomiting is defined as retching and expulsion of the stomach's contents through the mouth accompanied by shivering and salivation. $^{5}$ A previous study indicated that these two symptoms affected up to $70 \%$ of children undergoing chemotherapy. ${ }^{6}$ This prevalence is similar to that in adult patients with cancer, ranging from $60 \%$ to $72 \% .^{78}$

The aetiology of nausea and vomiting in children with cancer is multifactorial, ${ }^{9}$ but it is generally believed to be largely related to chemotherapy, which is the most common type of treatment for childhood cancer. ${ }^{5}$ Chemotherapeutic agents circulating in the blood stimulate the chemoreceptor trigger zone and result in acute nausea and 
vomiting. ${ }^{10}$ Likewise, repeated cycles of chemotherapy induce anticipatory nausea and vomiting, which is a learnt response to the treatment due to classical conditioning. ${ }^{11}$ Another cause of nausea and vomiting is haematopoietic stem cell transplantation, which is a common treatment for children who have malignancy with bone marrow failure. ${ }^{12}$ This can be explained by the gastroparesis that results from the administration of high-dose chemotherapy before stem cell transplantation. ${ }^{13}$ Radiotherapy can also lead to nausea and vomiting, but it depends on the treatment sites. ${ }^{14}$ Children who undergo radiotherapy in the upper abdominal region are at increased risk of nausea and vomiting because of the inflammatory substances released from the damaged gastrointestinal tissues. ${ }^{15}$ Those exposed to whole-brain radiation are similarly at risk because of increased intracranial pressure due to tissue swelling. ${ }^{16}$ Other causes of nausea and vomiting include anaesthesia given for surgery, ${ }^{17}$ analgesics (particularly opioids) ${ }^{18}$ and anxiety and emotional responses. ${ }^{5}$

Nausea and vomiting cause serious disruptions in patients' daily function and quality of life during cancer treatment. ${ }^{3-5}$ These two symptoms can also contribute to additional clinical problems, such as weight loss and cachexia. ${ }^{19}$ Such clinical problems have adverse effects on patients' general condition, interfering with their ability to continuously receive chemotherapy ${ }^{20}$; this, in turn, lowers the dose that can be administered ${ }^{21}$ and can even delay the overall cancer treatment. ${ }^{22}$ Given such devastating impacts, interventions that can alleviate nausea and vomiting are crucial, particularly for children with cancer. ${ }^{23} 24$ The most effective antiemetics are currently $5-\mathrm{HT}_{3}$ inhibitors, followed by $\mathrm{NK}_{1}$ inhibitors and neuroleptic drugs. ${ }^{25}$ Nevertheless, pharmacological intervention alone is insufficient to manage nausea and vomiting, with $>40 \%$ of children still experiencing nausea and vomiting after receiving antiemetics. ${ }^{26}$

\section{Description of the interventions}

Limitations of pharmacological treatment have led to an increase in the use of complementary and complementary medicine (CAM) to manage nausea and vomiting. ${ }^{27}$ According to the National Institutes of Health, ${ }^{28}$ CAM refers to medical products and practices that are not regarded as part of standard medical care. The types of CAM most commonly used to control nausea and vomiting among children with cancer include acupressure, ${ }^{26} 2930$ acupuncture ${ }^{31}$ hypnotherapy, ${ }^{32} 33$ aromatherapy ${ }^{34} 35$ and creative arts therapy. ${ }^{36}$

\section{How the interventions might work Acupressure}

Acupressure originated from traditional Chinese medicine and is used to regulate the basal energy inside the body through stimulation of acupoints via pressure.[37] The P6 acupoint is located on the ventral surface of the wrist and is considered to have antiemetic action. ${ }^{37}$

\section{Acupuncture}

Acupuncture also stems from traditional Chinese medicine and shares the same principle as acupressure. However, instead of applying pressure, acupuncture stimulates acupoints by insertion of fine needles into the body. ${ }^{29}$

\section{Hypnotherapy}

Hypnotherapy is a therapeutic technique used to produce changes in people's perception and cognition. ${ }^{38}$ Hypnotherapy encompasses different methods, among which relaxation and imagination are the most commonly used in children with cancer. ${ }^{32}{ }^{33}$ Through these methods, hypnotherapy helps people to achieve a deep state of psychological relaxation. ${ }^{39}$ When under a state of deep relaxation, people can much more easily override automatic thoughts; ${ }^{40}$ these include anticipatory nausea and vomiting, which are learned responses to cancer treatment. ${ }^{11}$ Likewise, hypnosis can decrease sympathetic tone, which helps minimize the symptoms associated with sympathetic arousal, such as nausea and vomiting. ${ }^{41}$

\section{Aromatherapy}

Aromatherapy refers to the use of essential oils, which are volatile liquids extracted from plants using streams and/or pressure. ${ }^{42}$ Inhalation of these volatile molecules stimulates olfactory receptors to produce nerve impulses, which are transmitted along the olfactory nerve to reach the amygdala and limbic system, ${ }^{43}$ interacting with the neuropsychological pathway to reduce nausea and vomiting. ${ }^{44}$

\section{Creative arts therapy}

Creative arts therapy is a broad term encompassing different treatment modalities such as dance, music, art and drama. ${ }^{36}$ It aims to facilitate people's emotional expression and improve their coping skills, ${ }^{36}{ }^{45}$ thus minimising the perceived severity of nausea and vomiting during cancer treatment.

\section{Why this review is important}

In 2017, the Multinational Association of Supportive Care in Cancer reiterated the importance of managing nausea and vomiting among children with cancer. They also issued a guideline to provide a consensus for healthcare professionals on how these two symptoms can be managed using a pharmacological approach. ${ }^{6}$ However, these two symptoms remain a significant clinical concern for children with cancer. ${ }^{5}$ This concern might be due to healthcare professionals' perception that nausea and vomiting are solely biological problems as well as the heavy reliance of these professionals on pharmacological treatment for symptom management. ${ }^{46}$ This point is particularly true because anticipatory nausea and vomiting are learnt responses to cancer and are difficult to control by medications alone. ${ }^{47}$ Increasingly more studies are demonstrating the effectiveness of different types of CAM to control nausea and vomiting during cancer treatment. ${ }^{27}$ Appropriate application of CAM enhances the 
effectiveness of antiemetics, thus reducing the symptom burden on children as well as improving their general condition and quality of life during cancer treatment.

\section{Objective}

The objective of this review is to identify, analyse and synthesise research evidence of the effectiveness of CAM on treatment-related nausea and vomiting in children with cancer.

\section{METHODS AND ANALYSIS}

The systematic review will start in August 2019, and the planned end date is December 2020.

\section{Patient and public involvement}

No patient involved.

\section{Criteria for considering studies for this review \\ Types of studies}

To be included in this review, the article must be a fully published randomised trial. The trial can either adopt a parallel control design or a crossover design.

\section{Types of participants}

We will include all studies of patients aged $\leq 18$ years who were diagnosed with any type of cancer and were undergoing active treatment for any type of cancer. However, we will exclude studies that involved patients receiving palliative treatment. Among studies of patients with diagnoses other than cancer, only those in which $\geq 50 \%$ of the patients had diagnoses of cancer will be included in this review.

\section{Types of interventions}

Any type of CAM will be included in this review. However, interventions involving dietary supplements (eg, ginger powder or vitamins) to reduce nausea and vomiting will be excluded.

Types of outcome measures

\section{Primary outcomes}

- Nausea, assessed by self-reported and objective measures.

- Vomiting, assessed by self-reported and objective measures.

\section{Secondary outcomes}

- Compliance and adherence to the intervention.

- Any adverse event is defined as an unfavourable and unintended sign or symptom that occurs during the use of CAM but is not necessarily caused by it. ${ }^{48}$ The most commonly reported adverse events of CAM include but are not limited to worsening symptoms, irritability, soreness, headache, pain, restlessness, sleep disturbance, infection, and tissue damage. ${ }^{49} 50$ To qualify as a reported adverse event, an incident must be explicitly referred to as an adverse event in the study. Health-related dropouts during the intervention period will also be counted as adverse events. ${ }^{51}$

\section{Search method for study identification}

The search will be conducted from 1 August 2019 to 30 September 2019. We will search the following 10 databases to identify relevant trials for this systematic review: MEDLINE, the Cochrane Central Register of Controlled Trials, EMBASE, CINAHL, PsycINFO, LILACS, OpenSIGLE, the Chinese Biomedical Literature Database, the Chinese Medical Current Contents and the Chinese National Knowledge Infrastructure. An expanded search will also be conducted to identify additional trials for review; for this expanded search, we will review the reference list of each selected article. The following four websites will also be searched: WHO International Clinical Trials Registry Platform (www.who.int/ictrp/en), Current Controlled Trials (www.controlled-trials.com), CenterWatch (www.centerwatch.com) and ClinicalTrials. gov (www.clinicaltrials.gov). A full electronic search strategy for one database, that is, Medline has been shown in the supplementary file.

\section{Data collection and analysis \\ Selection of studies}

The eligibility of each study will be independently assessed by two authors (KYH and WHCL) using a standardised form. A third author (GCFC), who is a senior researcher, will be consulted if the disagreement cannot be resolved by discussion. When multiple publications that belong to the same study are found, all of these publications will be marked with the same study identifier and all available data will be used.

\section{Data extraction and management}

To comply with the standards outlined in the Methodological Expectations of Cochrane Intervention Reviews, ${ }^{52}$ two authors will independently perform the data extraction using the data collection form for randomised controlled trials developed by Cochrane.$^{53}$ For each selected trial, the following information will be extracted:

1. Characteristics of the studies

- Sponsorship and authors' affiliations.

- Study design.

- Method of generating random sequences.

- Method of allocation concealment.

- Degree of masking.

- Any exclusion after randomisation.

- Loss to follow-up.

- Compliance.

- Selective outcome reporting.

2. Characteristics of the study population

- Country in which the trial was conducted.

- Inclusion and exclusion criteria for each trial.

- Number of subjects in each arm.

- Demographic characteristics, including sex, age, educational attainment, ethnicity, socioeconomic status and religious beliefs.

- Clinical characteristics, including type of cancer, cancer stage, age at diagnosis, time since diagnosis, 
primary or secondary cancer, treatment modality and comorbidities.

3. Details of the intervention

- Types of interventions.

- Frequency, duration, total number of sessions and format (ie, on an individual or group basis).

- Details of the control group.

- Adherence.

- Contamination.

4. Characteristics of outcomes

- Self-reported measures.

- Objective data.

- Length of time between end of the intervention and outcome measurement.

- Adverse events reported.

Any disagreement or ambiguity will be resolved through discussion with a third author (GCFC), who is a senior researcher in cancer care and symptom management. We will also contact the authors of each trial that was published within the last 10 years to seek clarification and obtain additional information or missing data. Similar data for each outcome will be extracted, if possible. If the trial contains multiple time points for primary outcomes, the outcome measured at the longest time interval will be selected for analysis. There is a well-established classification for chemotherapy-induced nausea and vomiting. ${ }^{13}$ Therefore, in the subgroup analysis of the effect of CAM on chemotherapy-induced nausea and vomiting, we will analyse the data according to three time points: anticipatory (within 24 hours prior to chemotherapy administration), acute (occurring within 24 hours of chemotherapy administration) and delayed (occurring 24 hours to 7 days after chemotherapy administration). ${ }^{13} 54$ Only adverse events that occurred during the intervention period will be included in the analysis. Adverse events that occurred during the follow-up (non-intervention) period will be excluded ${ }^{51}$ All data will be entered and combined using Review Manager 5.3 (Cochrane Collaboration, Nordic Cochrane Centre, Copenhagen, Denmark). The data entry process will involve two researchers: one (ATC) will enter the data into the software, and another (KKWL) will verify the entered data.

\section{Assessment of risk of bias in included studies}

The risk-of-bias assessment will follow the guideline in the Cochrane Handbook for Systematic Reviews of Intervention $^{55}$ and that in the Cochrane Consumers and Communication Review Group. ${ }^{56}$ For randomised controlled trials, the following domains will be assessed:

- Sequence generation.

- Allocation concealment.

- Blinding of participants and personnel.

- Blinding of outcome.

- Selective outcome reporting.

- Incomplete outcome data.

- Other sources of bias.

For the randomised trials that adopted a crossover design, the assessment will be extended to cover additional domains such as receiving the intervention in random order and evaluation of the carryover effect. ${ }^{556}$

Two authors (KYH and WHCL) will independently assess the risk of bias of the included studies. They will evaluate the degree of risk (ie, low, high or unclear) according to the criteria established by Higgins and Green.$^{55}$ Comments from a third author (GCFC, a senior researcher) will be sought when disagreement occurs. The risk of bias of all included trials will be presented in a figure.

\section{Measure of treatment effect}

Review Manager 5.3 will be used to conduct a meta-analysis. We will measure the treatment effects using ORs for dichotomous outcomes and the mean difference (MD) and standardised mean difference (SMD) for continuous outcomes. The $95 \%$ CIs will be reported for all outcomes.

\section{Units of analysis \\ Cluster randomization}

The guideline stipulated in the Cochrane Handbook of Systematic Reviews of Interventions will be followed to manage the data from cluster randomised controlled trials. ${ }^{55}$

First, we will check whether adequate adjustments have been made to account for clustering before estimating the treatment effects. If adequate adjustments have not been made, we will extract the data and estimate the treatment effect using a parallel control design, but we will adjust the standard errors to overcome the clustering effect. However, this analysis requires information on an appropriate intraclass correlation coefficient (ICC). Hence, we will contact the authors. If we fail to receive a response from the authors, we will estimate the ICC from the existing database or from the studies included in this review. If we are still unable to calculate an appropriate ICC, a sensitivity analysis will be conducted using a high ICC of 0.10, a moderate ICC of 0.01 and a low ICC of 0.00 . We will use Review Manager 5.3 to combine the estimates obtained from clustered randomised controlled trials with those from studies with a parallel control design.

\section{Crossover design}

If a trial has adopted a crossover design, we will only use the data collected before the first crossover time point to avoid any possible carryover effect. If the trial did not report these data, we will contact the corresponding author. If we do not receive a response from the author, this trial will be excluded from our meta-analysis and its findings will only be reported narratively.

\section{Missing data}

We will handle missing data in accordance with the guideline stipulated in the Cochrane Handbook for Systematic Reviews of Intervention. ${ }^{55}$ In particular, the following methods will be used:

- Contact the corresponding author to request missing data.

- Perform analysis of available cases.

- Address the potential impact of missing data on the findings of the review in the Discussion section. 


\section{Assessment of heterogeneity}

For clinical heterogeneity, we will examine the distribution of the following factors:

- Age.

- Sex.

- Diagnosis.

- Treatment modality.

Any uneven distribution that is unexpected will be addressed in the review in detail. With respect to statistical heterogeneity, we will compute the $I^{2}$ statistic, which is an index to quantify the percentage of variance in a meta-analysis that is attributable to study heterogeneity rather than sampling error. ${ }^{57} 58$ The $\chi^{2}$ test will also be conducted to examine the genuineness of that heterogeneity.

\section{Assessment of reporting biases}

We will strictly follow the search procedure described in this protocol (see Search method for study identification) to minimise any reporting bias. If we identify a sufficient number of studies (ie, $\geq 10$ ), we will graphically examine the publication bias using funnel plots. According to the guideline stipulated in the Cochrane Handbook for Systematic Reviews of Interventions, we will apply the test proposed by Egger et a $\tilde{l}^{9}$ to assess the funnel plot symmetry.

\section{Data synthesis}

The Cochrane Collaboration's statistical software (Review Manager 5.3) will be used for data synthesis. The data will be pooled for the meta-analysis when the included studies are sufficiently homogeneous with respect to subjects, interventions and outcomes. All similar studies will be pooled for a random-effects model to obtain the pooled intervention effect. The pooled intervention effect will be expressed in terms of the MD and $95 \%$ of CI if the outcome was reported as a continuous variable. If different scales were used to assess the outcome, the SMD will be used. If the outcome was measured as a dichotomous variable, we will convert the OR into the SMD as long as the underlying continuous measure followed an approximately normal distribution.

\section{Subgroup analysis and investigation of heterogeneity}

The following subgroup analyses will be performed for the primary outcomes if sufficient trials are available:

- Diagnosis.

- Stage of cancer.

If possible, we will also conduct a subgroup analysis to determine the effectiveness of CAM on nausea and vomiting caused by different cancer treatments (ie, chemotherapy and radiation). Because there is a clear classification for chemotherapy-induced nausea and vomiting, ${ }^{13}{ }^{54}$ the primary outcomes for this subgroup analysis will be defined by the following time points:

- Anticipatory (within 24 hours prior to chemotherapy administration).
- Acute (occurring within 24 hours of chemotherapy administration).

- Delayed (occurring 24 hours to 7 days after chemotherapy administration).

\section{Sensitivity analysis}

If possible, a sensitivity analysis will be carried out to examine the impact of including low-quality studies in the meta-analysis. Studies with a high or unclear risk of bias will be excluded to check the robustness of the conclusions.

\section{Ethics and dissemination}

The results will be presented at international conferences and published in peer-reviewed journals. As no individual data will be involved in this review, ethical approval is not required.

\section{DISCUSSION}

During the past few decades, different complementary and alternative therapies have been applied to manage nausea and vomiting associated with active treatment among children with cancer. ${ }^{26}{ }^{29-36}$ Nevertheless, no systematic review has yet been performed to compare the effectiveness of these therapies. We will fill this knowledge gap in the existing literature by conducting a systematic review on current evidence. The results are expected to assist healthcare professionals in their clinical decision making, particularly in relation to which complementary and alternative therapies are the most effective for management of nausea and vomiting during cancer treatment.

Acknowledgements The authors thank Angela Morben, DVM, ELS, from Edanz Group (www.edanzediting.com/ac), for editing a draft of this manuscript.

Contributors KYH, WHCL, KKWL, WX, ATC, LLKH, SYC, GCFC and JOKC: conceived and designed the study. KYH and WHCL: drafted the manuscript. KYH, WHCL and KKWL: critically revised the manuscript for important intellectual content. All authors approved the final version of the manuscript and agreed to be accountable for all aspects of the work.

\section{Funding Self-financed.}

Competing interests No, there are no competing interests for any author. Patient consent for publication Not required.

Provenance and peer review Not commissioned; externally peer reviewed. Data availability statement No data are available.

Open access This is an open access article distributed in accordance with the Creative Commons Attribution Non Commercial (CC BY-NC 4.0) license, which permits others to distribute, remix, adapt, build upon this work non-commercially, and license their derivative works on different terms, provided the original work is properly cited, appropriate credit is given, any changes made indicated, and the use is non-commercial. See: http://creativecommons.org/licenses/by-nc/4.0/.

ORCID iD

Ho Cheung William LI http://orcid.org/0000-0002-3195-7695

\section{REFERENCES}

1 World Health Organization. Global initiative for childhood cancer, 2014. Available: https://www.who.int/cancer/childhood-cancer/en/ [Accessed 24 Jan 2019].

2 Dupuis LL, Ethier M-C, Tomlinson D, et al. A systematic review of symptom assessment scales in children with cancer. BMC Cancer 2012;12:430 
3 HC L, Williams PD, Lopez V, et al. Relationships among therapyrelated symptoms, depressive symptoms, and quality of life in Chinese children hospitalized with cancer: an exploratory study. Cancer Nurs 2013:36:346-54.

4 Xia W, Li HCW, Lam KWK, et al. The impact of hematologic cancer and its treatment on physical activity level and quality of life among children in mainland China. Cancer Nurs 2018:1 (Online First: 14 November 2018).

5 Ruggiero A, Rizzo D, Catalano M, et al. Acute chemotherapy-induced nausea and vomiting in children with cancer: still waiting for a common consensus on treatment. J Int Med Res 2018;46:2149-56.

6 Dupuis LL, Sung L, Molassiotis A, et al. Updated MASCC/ESMO consensus recommendations: prevention of acute chemotherapyinduced nausea and vomiting in children. Support Care Cancer 2016;2017:323-31.

7 Navari RM. Management of chemotherapy-induced nausea and vomiting in pediatric patients. Pediatr Drugs 2017;19:213-22.

8 Navari RM, Aapro M. Antiemetic prophylaxis for chemotherapyinduced nausea and vomiting. N Engl J Med 2016;374:1356-67.

9 Rowland P. Management of nausea and vomiting in the pediatric blood and marrow transplant setting - are we covering all of our bases? A nursing perspective. Biol Blood Marrow Transplant 2011;17.

10 O'Brien C, O' Brien C. Nausea and vomiting. Can Fam Physician 2008:54:861-3.

11 Roscoe JA, Morrow GR, Aapro MS, et al. Anticipatory nausea and vomiting. Support Care Cancer 2011;19:1533-8.

12 Spencer GD, Hackman RC, McDonald GB, et al. A prospective study of unexplained nausea and vomiting after marrow transplantation. Transplantation 1986;42:602-7.

13 Schnell FM. Chemotherapy-Induced nausea and vomiting: the importance of acute antiemetic control. Oncologist 2003;8:187-98.

14 American Cancer Society. Radiation therapy-related nausea and vomiting, 2017. Available: https://www.cancer.org/treatment/ treatments-and-side-effects/physical-side-effects/nausea-andvomiting/risk-with-radiation.html [Accessed cited 21 January 2019].

15 Habibi M, Namimoghadam A, Korouni R, et al. Radiation-Induced nausea and vomiting: is $\mathrm{ABO}$ blood group as important as radiation and patient-related factors? an observational study. Medicine 2016;95:e4334

16 Texas Oncology. Radiation therapy for brain tumors., 2019. Available: https://www.texasoncology.com/types-of-cancer/brain-cancer/ radiation-therapy-for-brain-tumors [Accessed cited 23 January 2019].

17 Horn CC, Wallisch WJ, Homanics GE, et al. Pathophysiological and neurochemical mechanisms of postoperative nausea and vomiting. Eur J Pharmacol 2014;722:55-66.

18 Smith HS, Smith JM, Seidner P. Opioid-Induced nausea and vomiting. Ann Palliat Med 2012;1:121-9.

19 Sánchez-Lara K, Ugalde-Morales E, Motola-Kuba D, et al. Gastrointestinal symptoms and weight loss in cancer patients receiving chemotherapy. Br J Nutr 2013;109:894-7.

20 Alafafsheh A, Ahmad M. Tool development to assess nausea and vomiting among patients receiving chemotherapy. Int J Cancer Oncol 2016;3:1-5

21 Molassiotis A, Coventry PA, Stricker CT, et al. Validation and psychometric assessment of a short clinical scale to measure chemotherapy-induced nausea and vomiting: the MASCC antiemesis tool. J Pain Symptom Manage 2007;34:148-59.

22 Osoba D, Zee B, Warr D, et al. Quality of life studies in chemotherapy-induced emesis. Oncology 1996;53:92-5.

23 Dupuis LL, Boodhan S, Holdsworth M, et al. Guideline for the prevention of acute nausea and vomiting due to antineoplastic medication in pediatric cancer patients. Pediatr Blood Cancer 2013;60:1073-82.

24 Jordan K, Roila F, Molassiotis A, et al. Antiemetics in children receiving chemotherapy. MASCC/ESMO guideline update 2009. Support Care Cancer 2011;19:37-42.

25 Brafford MV, Glode A. Olanzapine: an antiemetic option for chemotherapy-induced nausea and vomiting. J Adv Pract Oncol 2014;5:24-9.

26 Yeh CH, Chien L-C, Chiang YC, et al. Reduction in nausea and vomiting in children undergoing cancer chemotherapy by either appropriate or sham auricular acupuncture points with standard care. $J$ Altern Complement Med 2012;18:334-40.

27 Zeltzer LK, Dolgin MJ, LeBaron S, et al. A randomized, controlled study of behavioral intervention for chemotherapy distress in children with cancer. Pediatrics 1991;88:34-42.

28 National Institutes of Health. Complementary and alternative medicine, 2018. Available: https://www.nlm.nih.gov/tsd/acquisitions/ cdm/subjects24.html [Accessed 21 Jan 2019].
29 Dupuis LL, Kelly KM, Krischer JP, et al. Acupressure bands do not improve chemotherapy-induced nausea control in pediatric patients receiving highly emetogenic chemotherapy: a single-blinded, randomized controlled trial. Cancer 2018;124:1188-96.

30 Jones E, Isom S, Kemper KJ, et al. Acupressure for chemotherapyassociated nausea and vomiting in children. $J$ Soc Integr Oncol 2008:6:141-5.

31 Reindl TK, Geilen W, Hartmann R, et al. Acupuncture against chemotherapy-induced nausea and vomiting in pediatric oncology. interim results of a multicenter crossover study. Support Care Cancer 2006;14:172-6.

32 Hawkins PJ, Liossi C, Ewart BW, et al. Hypnotherapy for control of anticipatory nausea and vomiting in children with cancer: preliminary findings. Psychooncology 1995;4:101-6.

33 Jacknow DS, Tschann JM, Link MP, et al. Hypnosis in the prevention of chemotherapy-related nausea and vomiting in children. $J$ Dev Behav Pediatr 1994;15:258-64.

34 Evans A, Malvar J, Garretson C, et al. The use of Aromatherapy to reduce chemotherapy-induced nausea in children with cancer: a randomized, double-blind, placebo-controlled trial. J Pediatr Oncol Nurs 2018;35:392-8.

35 Ndao DH, Ladas EJ, Cheng B, et al. Inhalation aromatherapy in children and adolescents undergoing stem cell infusion: results of a placebo-controlled double-blind trial. Psychooncology 2012;21:247-54.

36 Madden JR, Mowry P, Gao D, et al. Creative arts therapy improves quality of life for pediatric brain tumor patients receiving outpatient chemotherapy. J Pediatr Oncol Nurs 2010;27:133-45.

37 Lee J, Dodd M, Dibble S, et al. Review of acupressure studies for chemotherapy-induced nausea and vomiting control. J Pain Symptom Manage 2008;36:529-44.

38 Jensen MP, Jamieson GA, Lutz A, et al. New directions in hypnosis research: strategies for advancing the cognitive and clinical neuroscience of hypnosis. Neurosci Conscious 2017;3:1-14.

39 Vickers A, Zollman C, Payne DK. Hypnosis and relaxation therapies. West J Med 2001;175:269-72.

40 Oakley DA, Halligan PW. Hypnotic suggestion and cognitive neuroscience. Trends Cogn Sci 2009;13:264-70.

41 Goldstein LH, Berkovitch M. Nausea and vomiting in pregnancy. In: Schaefer C, Peter P, Miller RK, eds. Drugs during pregnancy and lactation: treatment options and risk assessment. Amsterdam, Boston: Elsevier Academic Press, 2007: 78-93.

42 Ali B, Al-Wabel NA, Shams S, et al. Essential oils used in aromatherapy: a systemic review. Asian Pac J Trop Biomed 2015;5:601-11.

43 Sowndhararajan K, Kim S. Influence of Fragrances on human psychophysiological activity: with special reference to human electroencephalographic response. Sci Pharm 2016;84:724-51.

44 Lis-Balchin M. Essential oils and 'aromatherapy': their modern role in healing. J R Soc Health 1997;117:324-9.

45 Puig A, Lee SM, Goodwin L, et al. The efficacy of creative arts therapies to enhance emotional expression, spirituality, and psychological well-being of newly diagnosed stage I and stage II breast cancer patients: a preliminary study. Arts Psychother 2006;33:218-28.

46 Molassiotis A, Fernández-Ortega P, Pud D, et al. Use of complementary and alternative medicine in cancer patients: a European survey. Ann Oncol 2005;16:655-63.

47 Kamen C, Tejani MA, Chandwani K, et al. Anticipatory nausea and vomiting due to chemotherapy. Eur J Pharmacol 2014;722:172-9.

48 Zorzela L, Boon H, Mior S, et al. Serious adverse events associated with pediatric complementary and alternative medicine. Eur J Integr Med 2014;6:467-72.

49 Humphreys BK. Possible adverse events in children treated by manual therapy: a review. Chiropr Osteopat 2010;18.

50 Xu S, Wang L, Cooper E, et al. Adverse events of acupuncture: a systematic review of case reports. Evid Based Complement Alternat Med 2013;2013:581203.

51 Cui H, Wang Q, Pedersen M, et al. The safety of tai chi: a metaanalysis of adverse events in randomized controlled trials. Contemp Clin Trials 2019;82:85-92.

52 Cochrane Community. Methodological expectations of Cochrane intervention reviews (MECIR), 2018. Available: https://community. cochrane.org/mecir-manual [Accessed 3 Jul 2019].

53 Cochrane. Data collection form (for RCTs), 2019. Available: https:// training.cochrane.org/data-collection-form-rcts [Accessed 3 July 2019].

54 Dupuis LL, Robinson PD, Boodhan S, et al. Guideline for the prevention and treatment of anticipatory nausea and vomiting due to chemotherapy in pediatric cancer patients. Toronto: Pediatric Oncology Group of Ontario, 2014: 61. 1506-12. 
55 Higgins JPT, Green S. Cochrane Handbook for systematic reviews of interventions version 5.1.0, 2011. https://www.cochrane-handbook. org

56 Ryan R, Santesso N, Hill S, et al. Consumer-oriented interventions for evidence-based prescribing and medicines use: an overview of systematic reviews. Cochrane Database Syst Rev 2011;5.
57 Higgins JPT, Thompson SG. Quantifying heterogeneity in a metaanalysis. Stat Med 2002;21:1539-58.

58 Higgins JPTet al. Measuring inconsistency in meta-analyses. BMJ 2003;327:557-60.

59 Egger M, Smith GD, Schneider M, et al. Bias in meta-analysis detected by a simple, graphical test. BMJ 1997;315:629-34. 